\title{
Resposta fenotípica de arroz de terras altas ao estresse de fósforo no solo
}

\author{
Cleber M. Guimarães ${ }^{1}$, Luís F. Stone ${ }^{1} \&$ Péricles C. F. Neves ${ }^{1}$
}

\section{RESUMO}

Avaliaram-se, neste trabalho, em condições de baixo e alto teor de fósforo $(\mathrm{P})$ no solo, 51 linhagens de arroz provenientes do Banco Ativo de Germoplasma da Embrapa Arroz e Feijão, com o objetivo de identificar indicadores de eficiência no uso de $\mathrm{P}$ e linhagens com eficiência diferenciada no uso desse nutriente. O índice de eficiência do uso de fósforo mostrou ser mais adequado que a produtividade em baixo teor de fósforo no solo, na avaliação da eficiência diferenciada das linhagens no uso do P. Três linhagens, CNA 4634, CNA 4120 e CNA 3178, apresentam bom comportamento produtivo, tanto em alto como em baixo teor de $\mathrm{P}$ no solo e 22 mostraram bom comportamento produtivo em alto teor de $\mathrm{P}$ no solo; entretanto, não foram eficientes no aproveitamento de baixo $\mathrm{P}$ disponível no solo enquanto as demais foram desconsideradas em razão de indicarem baixo comportamento produtivo sob boa disponibilidade de P.

Palavras-chave: Oryza sativa L., índice de eficiência do uso de fósforo, cultivares eficientes e não eficientes

\section{Response of upland rice in soils with low content of phosphorus}

\begin{abstract}
Fifty-one lines from Embrapa Rice \& Beans Germplasm Bank were evaluated under conditions of low and high soil phosphorus $(\mathrm{P})$ content, in order to identify $\mathrm{P}$ use efficiency indicators as well as lines with differentiated response to $\mathrm{P}$ use efficiency. Phosphorus use efficiency index was more suitable to evaluate lines in relation to $\mathrm{P}$ use efficiency than grain yield at low soil P content. Three lines, CNA 4634, CNA 4120, and CNA 3178 showed good grain yield performance at high as well as at low soil P content, while 22 lines showed good grain yield performance at high soil P content, but were not efficient in the use of low available soil $\mathrm{P}$, while the rest were not considered because they showed low yield performance at high soil $\mathrm{P}$ content.
\end{abstract}

Key words: Oryza sativa L., phosphorus use efficiency index, P-efficient and non-efficient cultivars 


\section{INTRODUÇÃO}

A deficiência de fósforo é considerada um dos principais fatores que limitam a produtividade do arroz em muitos solos da região do Cerrado (Fageria, 1982), agravada quando ocorre não só a baixa concentração do elemento no solo como, também, a intensa adsorsão às partículas do solo (Sanchez \& Salinas, 1981; Fageria \& Baligar, 1997; Dobermann et al., 1998). Esses autores estimaram que mais de $90 \%$ do total de fósforo aplicado no solo podem ser rapidamente transformados em formas não prontamente disponíveis. Considerando-se esses aspectos, a identificação de germoplasma capaz de utilizar os nutrientes menos disponíveis no solo se constitui em importante estratégia de baixo custo para promover a sustentabilidade da agricultura das regiões marginalizadas (Otani \& Ae, 1996). Esta estratégia foi confirmada por Wissuwa \& Ae (2001) ao transferirem QTL de alta capacidade de absorção de P, da cultivar 'Kasalath', tolerante à deficiência de $\mathrm{P}$, para a cultivar moderna de arroz ' $\mathrm{Ni}$ pponbare’, com alto índice de colheita, o que triplicou sua produtividade em condições de baixa disponibilidade de P. Um sistema radicular bem desenvolvido, capaz de explorar grande volume de solo, é reconhecido como um dos mais significativos mecanismos de adaptação da planta para garantir uma absorção suficiente de P (Sattelmacher et al., 1994; Horst et al., 2001); por outro lado, a arquitetura radicular das plantas pode sofrer alterações em resposta à deficiência de fósforo. Tem-se observado aumento no crescimento radicular lateral e nas ramificações secundárias laterais, em detrimento do alongamento da raiz primária do feijoeiro (Lynch \& Brown, 2001). Uma adaptação comumente constatada na maioria das espécies é o aumento do comprimento e da densidade dos pêlos absorventes que contribuem para o aumento da absorção de fósforo através da expansão da superfície radicular (Aboulroos \& Nielsen, 1979; Eissenstar, 1992; Gahoonia \& Nielsen, 1997; Lynch \& Brown, 2001). Wissuwa (2005) observou que a correlação entre a absorção de fósforo e o crescimento relativo das raízes sob condições de deficiência de $\mathrm{P}$ era maior que em ambiente de boa disponibilidade de fósforo porém Otani \& Ae (1996) mostraram que a absorção de P foi fortemente correlacionada com o comprimento radicular em solos com alta disponibilidade de P. Haynes \& Ludecke (1981) também não observaram relação entre a absorção de $\mathrm{P}$ e a massa radicular total em Lotus pedunculatus Cav mas se sabe que vários são os fatores que se interrelacionam e atuam conjuntamente no aumento da eficiência da absorção de $\mathrm{P}$, pois a quantidade adicional de $\mathrm{P}$ absorvida em função da maior eficiência externa das raízes, associada à morfologia e à fisiologia, contribuirá para o maior crescimento radicular (Wissuwa, 2003) e este, por sua vez, para o aumento da absorção do nutriente. Define-se como germoplasma eficiente, aquele que requer menos nutriente que o ineficiente para atingir patamares semelhantes de produtividade (Gourley et al., 1993).

Objetivou-se, com este trabalho, identificar indicadores da eficiência do uso de fósforo no solo pelo arroz, assim como linhagens com eficiência diferenciada no uso deste nutriente quando em baixa disponibilidade, porém com eficiência produtiva sob altas condições de $\mathrm{P}$.

\section{MATERIAL E MÉTODOS}

O estudo foi conduzido em condições de campo, no período das águas do ano agrícola de 2003-2004, na área experimental da Embrapa Arroz e Feijão, em Santo Antônio de Goiás, GO, de coordenadas geográficas $16^{\circ} 28^{\prime}$ de latitude sul, 49 $19^{\circ}$ ' de longitude oeste e altitude de $823,77 \mathrm{~m}$, em sua estação agroclimática, em Latossolo Vermelho distrófico, de textura argilosa. A análise química inicial dos solos na camada de 0-20 cm de profundidade, apresentou os seguintes resultados: $\mathrm{pH}\left(\mathrm{H}_{2} \mathrm{O}\right) 5,6 ; \mathrm{Ca}^{2+} 24,3 \mathrm{mmol}_{\mathrm{c}} \mathrm{dm}^{-3}$, $\mathrm{Mg}^{2+} 11,4 \mathrm{mmol}_{\mathrm{C}} \mathrm{dm}^{-3} ; \mathrm{Al}^{3+} 1,0 \mathrm{mmol}_{\mathrm{C}} \mathrm{dm}^{-3} ; \mathrm{P} 0,4 \mathrm{mg} \mathrm{dm}^{-3}$ e K $90 \mathrm{mg} \mathrm{dm}^{-3}$, determinadas segundo metodologia apresentada pela Claessen (1997). Avaliaram-se 51 linhagens de arroz (Oryza sativa L.) provenientes do Banco Ativo de Germoplasma da Embrapa Arroz e Feijão, em condições de baixo e alto teor de fósforo no solo, no delineamento de blocos ao acaso, com três repetições. As condições de fósforo no solo constituíram os experimentos, que foram submetidos a análise individual e conjunta, de variância. No tratamento de baixo teor de fósforo aplicaram-se 12, 48, 20 e $20 \mathrm{~kg} \mathrm{ha}^{-1}$ de $\mathrm{N}, \mathrm{K}_{2} \mathrm{O}$, FTE BR 12 e sulfato de zinco, respectivamente e, no tratamento de alto teor de fósforo, aplicou-se a mesma adubação acrescida de duas doses de fósforo, $600+$ $150 \mathrm{~kg} \mathrm{ha}^{-1}$ de $\mathrm{P}_{2} \mathrm{O}_{5}$, a primeira no ano agrícola 2002-03 e a segunda em 2003-04. Determinaram-se: a produtividade de grãos e os parâmetros agronômicos, a massa de matéria seca das plantas e dos grãos, o índice de colheita, a esterilidade de espiguetas, o número de grãos por panícula e a altura das plantas. A secagem da biomassa foi efetuada em estufa com circulação interna forçada de ar, a $70^{\circ} \mathrm{C}$, até massa constante e, depois, pesada em uma balança de precisão; adotouse também o índice de susceptibilidade (Fisher \& Maurer, 1978) adaptado para a eficiência do uso de fósforo, conforme apresentado abaixo:

$$
\begin{gathered}
\mathrm{IP}=\left(\mathrm{Y}_{\mathrm{c} / \mathrm{P}}-\mathrm{Y}_{\mathrm{s} / \mathrm{P}}\right) /\left(\mathrm{Y}_{\mathrm{c} / \mathrm{P}} * \mathrm{D}\right) \\
\mathrm{D}=1-\left(\mathrm{X}_{\mathrm{s} / \mathrm{P}} / \mathrm{X}_{\mathrm{c} / \mathrm{P}}\right)
\end{gathered}
$$

em que: IP é o índice de eficiência do uso de fósforo, D é a severidade do estresse induzido pela baixa disponibilidade de $\mathrm{P}, \mathrm{Y}_{\mathrm{C} / \mathrm{P}}$ e $\mathrm{Y}_{\mathrm{S} / \mathrm{P}}$ são, respectivamente, as produtividades individuais das linhagens nos tratamentos de alto e baixo teor de $\mathrm{P}$ e $X_{c / P}$ e $X_{s / P}$ são, respectivamente, as produtividades médias de todas as linhagens nos tratamentos de alto e baixo teor de P. A produtividade é tanto menos afetada pelo nível de estresse induzido pela baixa disponibilidade de $\mathrm{P}$ quanto menor for o valor de IP. As linhagens foram divididas em quartis, conforme a média da produtividade sob condições de alta disponibilidade de $\mathrm{P}$ e a média do seu índice de eficiência do uso de $\mathrm{P}$. O quartil 1 foi definido pelas produtividades superiores 
à produtividade média em ambiente com alta disponibilidade de $\mathrm{P}\left(\mathrm{X}_{\mathrm{C} / \mathrm{P}}\right)$, e pelos IPs inferiores ao IP médio ( $\left.\mathrm{IP}_{\mathrm{X}}\right)$; o quartil 2 , pelas produtividades superiores a $X_{C / P}$ e IPs superiores ao $\mathrm{IP}_{\mathrm{x}}$; o quartil 3, pelas produtividades inferiores a $\mathrm{X}_{\mathrm{C} / \mathrm{P}}$ e IPs superiores ao $\mathrm{IP}_{\mathrm{x}}$ e, finalmente, o quartil 4, pelas produtividades inferiores a $\mathrm{X}_{\mathrm{c} / \mathrm{P}}$ e IPs inferiores ao $\mathrm{IP}_{\mathrm{x}}$.

\section{RESULTADOS E DISCUSSÃO}

Verificou-se grande variabilidade entre as linhagens de arroz de terras altas quanto ao comportamento em baixo e alto teor de fósforo no solo, conforme todos os parâmetros avaliados, como pode ser observado nas Tabelas 1, 2 e 3. Os tratamentos com fósforo afetaram, com intensidade diferenciada, a produtividade das linhagens, por se ter observado efeito significativo na interação nível de fósforo x linhagens; o mesmo ocorreu quanto ao acúmulo de matéria seca das plantas (Tabelas 1, 2 e 3). As linhagens produziram, em média, $1619 \mathrm{~kg} \mathrm{ha}^{-1}$ no tratamento com alto fósforo e $478 \mathrm{~kg} \mathrm{ha}^{-1}$ no tratamento com baixo fósforo, enquanto o acúmulo de matéria seca foi de 3046 e $2025 \mathrm{~kg} \mathrm{ha}^{-1}$, nos tratamentos com alto e baixo P, respectivamente. O nível de fósforo também afetou significativamente a esterilidade de espiguetas, o número de grãos por panícula e a altura das plantas (Tabelas 1 e 3); entretanto, a intensidade do efeito de P sobre esses componentes agronômicos foi semelhante em todas as linhagens, por não ter sido observado efeito significativo nas interações nível de fósforo x linhagens em cada um dos referidos componentes agronômicos. Fageria \& Baligar (1997) também mostraram efeito do nível de fósforo sobre o crescimento das plantas de arroz de terras altas, porém em solução nutritiva. A média da esterilidade de espiguetas foi 16,0 e 19,1\%, nos tratamentos com alto e baixo teor de P, respectivamente, enquanto as panículas produziram, em média, 115 e 83 grãos e as plantas apresentaram altura máxima, por ocasião da colheita, de 97 e $80 \mathrm{~cm}$ nos tratamentos com alto e baixo teor de fósforo, respectivamente. Verificou-se que o índice de eficiência do uso de fósforo se correlacionou significativamente com a produtividade de grãos, massa de 100 grãos, esterilidade de espiguetas e com o número de grãos por panícula em baixo teor de fósforo no solo; por outro lado a produti- vidade de grãos em baixo teor de fósforo se correlacionou com a esterilidade de grãos e com o número de grãos por panícula porém não se correlacionou com a massa de 100 grãos (Tabela 4). Considerando-se que esses componentes da produtividade, massa de 100 grãos, esterilidade de espiguetas e número de grãos por panícula, são influenciados pelo nível de P no solo (Fageria, 1999) e que a massa de 100 grãos não se correlacionou com a produtividade do arroz de terras altas sob condições de baixa disponibilidade de $\mathrm{P}$ no solo, este não pode ser considerado um indicador confiável da eficiência do uso do P no solo pelo arroz de terras altas; fato explicado em razão da produtividade em baixo P não expressar apenas o efeito da disponibilidade do P no solo, enquanto o IP expressa o diferencial relativo de produtividade das linhagens em condições de alta e baixa disponibilidade de $\mathrm{P}$ no solo, considerando-se também a pressão do estresse em que as linhagens foram avaliadas.

Na seleção das linhagens considerou-se a produtividade em alto $\mathrm{P}$, pois são desejáveis a divergência fenotípica na eficiência do uso do P quando em baixa disponibilidade no solo e a eficiência produtiva quando em alta disponibilidade. Conforme a distribuição das linhagens em quartis, dois grupos foram selecionados: o primeiro se compunha das linhagens CNA 4634, CNA 4120 e CNA 3178, caracterizadas por apresentarem produtividade acima de $1619 \mathrm{~kg} \mathrm{ha}^{-1}$ (média da produtividade das linhagens sem o estresse de baixa disponibilidade de $\mathrm{P}$ no solo) e IP inferior a 0,95 (média do índice de eficiência do uso de P), ou seja, apresentam bom comportamento produtivo tanto em alto como em baixo P no solo; o outro grupo das linhagens, CNA 4108, CNA 4141, CNA 4640, CNA 2721, CNA 4150, CNA 4476, CNA 4474, CNA 5171, CNA 4181, CNA 1462, CNA 4125, CNA 2042, CNA 1168, CNA 4178, CNA 5165, CNA 2720, CNA 5164, CNA 8521, CNA 4140, CNA 5166, CNA 4617 e CNA 4164, que produziram acima de $1619 \mathrm{~kg} \mathrm{ha}^{-1}$, porém IP superior a 0,95, isto é, apresentam bom comportamento produtivo em alto $\mathrm{P}$ no solo; entretanto, não são eficientes no aproveitamento de baixo P disponível no solo (Figura 1). Os outros quartis, por enquadrarem as linhagens que apresentam baixo comportamento produtivo sob boa disponibilidade de $\mathrm{P}$ no solo, não foram considerados.

Tabela 1. Resumo da análise de variância para os componentes agronômicos produtividade de grãos, massa da matéria seca das plantas (MS), índice de colheita (IC), massa de 100 grãos (MS100), esterilidade de espiguetas (EE), número de grãos por panícula (GPAN), altura das plantas e índice de eficiência do uso de fósforo no solo (IP)

\begin{tabular}{|c|c|c|c|c|c|c|c|c|c|}
\hline \multirow[b]{2}{*}{ Fonte de Variação } & \multirow[b]{2}{*}{ G.L. } & \multicolumn{8}{|c|}{ Quadrado Médio1 } \\
\hline & & $\begin{array}{l}\text { Produtividade } \\
\left(\mathrm{kg} \mathrm{ha}^{-1}\right)\end{array}$ & $\begin{array}{c}\text { MS } \\
\left(\mathrm{kg} \mathrm{ha}^{-1}\right)\end{array}$ & $\begin{array}{l}\text { IC } \\
(\%)\end{array}$ & $\begin{array}{l}\text { MS100 } \\
\text { (g) }\end{array}$ & $\begin{array}{l}\mathrm{EE} \\
(\%)\end{array}$ & $\begin{array}{l}\text { GPAN } \\
\left(N^{0}\right)\end{array}$ & $\begin{array}{l}\text { Altura } \\
\text { (cm) }\end{array}$ & IP \\
\hline Nível fósforo (P) & 1 & $99.574 .500,44 * \star *$ & $79,73^{\star *}$ & $51,50^{\text {ns }}$ & $0,06^{\mathrm{ns}}$ & $732,19 * \star$ & $76.099,39 * \star \star$ & $22.716,12^{\star \star *}$ & - \\
\hline Erro a & 4 & $1.222 .236,72$ & 1,70 & 71,70 & 0,21 & 27,91 & 598,76 & 233,12 & 0,03 \\
\hline Linhagens (L) & 50 & $306.566,87^{\star \star \star *}$ & $2,31^{\star * \star}$ & $212,04 * \star \star$ & $0,68^{\star \star *}$ & $185,57^{\star * *}$ & $2.009,85^{\star \star \star}$ & $494,23 * \star \star$ & $0,10^{\star \star \star}$ \\
\hline$P \times L$ & 50 & $285.530,18^{\star * *}$ & 0,64 ** & $67,66^{*}$ & $0,13^{\text {ns }}$ & $55,49^{\text {ns }}$ & $765,80^{\text {ns }}$ & $50,41^{\text {ns }}$ & - \\
\hline Erro b & 200 & $76.919,42$ & 0,37 & 47,34 & 0,09 & 41,08 & 720,31 & 62,64 & - \\
\hline CV (\%) & & 26,46 & 24,13 & 14,63 & 10,37 & 36,5 & 27,15 & 8,96 & 18,81 \\
\hline
\end{tabular}

1 ns $-F$ não-significativo a $5 \%$; * $-F$ significativo a $5 \%$; ${ }^{\star \star}$ - F significativo a $1 \% ;{ }^{\star \star \star}$ - F significativo a $0,1 \%$ 
Tabela 2. Produtividade, índice de eficiência do uso de fósforo, índice de colheita e esterilidade de espiguetas das linhagens avaliadas para a eficiência ao uso de fósforo ${ }^{1}$

\begin{tabular}{|c|c|c|c|c|c|c|c|}
\hline \multirow{2}{*}{ Linhagens } & \multicolumn{2}{|c|}{ Produtividade $\left(\mathrm{kg} \mathrm{ha}^{-1}\right)$} & \multirow{2}{*}{$\mathrm{IP}^{2}$} & \multicolumn{2}{|c|}{ Índice de colheita (\%) } & \multicolumn{2}{|c|}{ Esterilidade Espiguetas (\%) } \\
\hline & C/fósforo & S/fósforo & & C/fósforo & S/fósforo & C/fósforo & S/tósforo \\
\hline CNA 2826 & $693 \mathrm{~b}$ & $406 \mathrm{~b}$ & $0,57 \mathrm{~b}$ & $17,18 \mathrm{~d}$ & $35,86 \mathrm{~b}$ & $44,46 \mathrm{a}$ & $32,83 \mathrm{a}$ \\
\hline CNA 4179 & $874 \mathrm{~b}$ & $419 \mathrm{~b}$ & $0,72 \mathrm{~b}$ & $54,70 \mathrm{a}$ & 54,06 a & $8,23 \mathrm{c}$ & $15,97 \mathrm{~b}$ \\
\hline CNA 2739 & $979 \mathrm{~b}$ & $228 \mathrm{c}$ & $1,07 \mathrm{a}$ & $45,62 \mathrm{~b}$ & $44,21 \mathrm{~b}$ & $9,05 \mathrm{c}$ & $11,91 \mathrm{~b}$ \\
\hline CNA 0486 & $1031 \mathrm{~b}$ & $411 \mathrm{~b}$ & $0,86 \mathrm{a}$ & $43,21 \mathrm{~b}$ & $45,15 \mathrm{~b}$ & $25,93 \mathrm{~b}$ & $26,38 \mathrm{a}$ \\
\hline CNA 2836 & $1054 \mathrm{~b}$ & $398 \mathrm{~b}$ & $0,87 \mathrm{a}$ & $31,07 \mathrm{c}$ & $33,59 \mathrm{~b}$ & $24,39 \mathrm{~b}$ & $17,46 \mathrm{~b}$ \\
\hline CNA 3373 & $1072 \mathrm{~b}$ & $473 \mathrm{a}$ & $0,68 \mathrm{~b}$ & $54,29 \mathrm{a}$ & $53,65 \mathrm{a}$ & $14,24 \mathrm{c}$ & $19,07 \mathrm{~b}$ \\
\hline CNA 4411 & $1076 \mathrm{~b}$ & $419 \mathrm{~b}$ & $0,84 \mathrm{a}$ & $37,96 \mathrm{c}$ & $45,07 \mathrm{~b}$ & $13,06 \mathrm{c}$ & $21,71 \mathrm{a}$ \\
\hline CNA 0799 & $1140 \mathrm{~b}$ & $327 \mathrm{c}$ & $0,98 \mathrm{a}$ & $41,23 \mathrm{c}$ & $47,69 \mathrm{a}$ & $21,72 b$ & $15,96 \mathrm{~b}$ \\
\hline CNA 4291 & $1166 \mathrm{~b}$ & $782 \mathrm{a}$ & $0,32 \mathrm{~b}$ & $37,57 \mathrm{c}$ & $55,42 \mathrm{a}$ & $30,92 \mathrm{~b}$ & $22,35 \mathrm{a}$ \\
\hline CNA 1383 & $1197 \mathrm{~b}$ & $588 \mathrm{a}$ & $0,69 \mathrm{~b}$ & $43,51 \mathrm{~b}$ & $51,08 \mathrm{a}$ & $20,14 \mathrm{c}$ & $17,36 \mathrm{~b}$ \\
\hline CNA 5163 & $1208 \mathrm{~b}$ & $658 \mathrm{a}$ & $0,56 \mathrm{~b}$ & 51,99 a & $45,24 \mathrm{~b}$ & $12,00 \mathrm{c}$ & $23,51 \mathrm{a}$ \\
\hline CNA 2523 & $1269 \mathrm{~b}$ & $424 \mathrm{~b}$ & $0,91 \mathrm{a}$ & $38,02 \mathrm{c}$ & $40,02 \mathrm{~b}$ & $23,67 \mathrm{~b}$ & $29,07 \mathrm{a}$ \\
\hline CNA 4171 & $1297 \mathrm{~b}$ & $501 \mathrm{a}$ & $0,87 \mathrm{a}$ & $51,96 \mathrm{a}$ & $50,64 \mathrm{a}$ & $16,24 \mathrm{c}$ & $12,63 \mathrm{~b}$ \\
\hline CNA 4148 & $1303 \mathrm{~b}$ & $578 \mathrm{a}$ & $0,73 \mathrm{~b}$ & $40,55 \mathrm{c}$ & $38,30 \mathrm{~b}$ & $16,78 \mathrm{c}$ & $21,53 \mathrm{a}$ \\
\hline CNA 6571 & $1325 \mathrm{~b}$ & $777 \mathrm{a}$ & $0,58 \mathrm{~b}$ & $54,38 \mathrm{a}$ & $43,85 \mathrm{~b}$ & $15,71 \mathrm{c}$ & $22,97 \mathrm{a}$ \\
\hline CNA 3289 & $1351 \mathrm{~b}$ & $290 \mathrm{c}$ & $1,10 \mathrm{a}$ & $46,14 \mathrm{~b}$ & $44,37 \mathrm{~b}$ & $12,87 \mathrm{c}$ & 19,98 b \\
\hline CNA 5021 & $1396 \mathrm{~b}$ & $478 \mathrm{a}$ & $0,93 \mathrm{a}$ & $60,53 \mathrm{a}$ & $52,40 \mathrm{a}$ & $13,25 \mathrm{c}$ & $14,72 \mathrm{~b}$ \\
\hline CNA 5167 & $1429 \mathrm{~b}$ & $527 \mathrm{a}$ & $0,89 \mathrm{a}$ & $47,62 \mathrm{~b}$ & $43,52 \mathrm{~b}$ & $8,25 \mathrm{c}$ & $23,14 \mathrm{a}$ \\
\hline CNA 4105 & $1449 \mathrm{a}$ & $448 \mathrm{~b}$ & $0,99 \mathrm{a}$ & $54,57 \mathrm{a}$ & $41,54 \mathrm{~b}$ & $14,14 \mathrm{c}$ & $17,23 \mathrm{~b}$ \\
\hline CNA 6564 & $1481 \mathrm{~b}$ & $440 \mathrm{~b}$ & $0,92 \mathrm{a}$ & $56,17 \mathrm{a}$ & $45,95 \mathrm{~b}$ & $7,37 \mathrm{c}$ & $20,20 \mathrm{~b}$ \\
\hline CNA 4166 & $1484 \mathrm{a}$ & $315 \mathrm{c}$ & $1,12 \mathrm{a}$ & $48,40 \mathrm{~b}$ & $45,65 \mathrm{~b}$ & $8,98 \mathrm{c}$ & $14,06 \mathrm{~b}$ \\
\hline CA 780284 & $1537 \mathrm{a}$ & $532 a$ & $0,91 \mathrm{a}$ & $40,73 \mathrm{c}$ & $40,10 \mathrm{~b}$ & $14,88 \mathrm{c}$ & $17,05 \mathrm{~b}$ \\
\hline CNA 4180 & $1538 \mathrm{a}$ & $412 \mathrm{~b}$ & $1,03 \mathrm{a}$ & 53,84 a & 49,49 a & $9,82 \mathrm{c}$ & $13,98 \mathrm{~b}$ \\
\hline CNA 4144 & $1553 \mathrm{a}$ & $521 \mathrm{a}$ & $0,94 \mathrm{a}$ & $34,42 \mathrm{c}$ & $42,25 \mathrm{~b}$ & $18,95 \mathrm{c}$ & $28,25 \mathrm{a}$ \\
\hline CNA 2865 & $1557 \mathrm{a}$ & $503 a$ & $0,95 \mathrm{a}$ & $44,94 \mathrm{~b}$ & $48,24 \mathrm{a}$ & $22,22 \mathrm{~b}$ & $19,83 \mathrm{~b}$ \\
\hline CNA 6430 & $1573 a$ & $432 \mathrm{~b}$ & $1,04 \mathrm{a}$ & $47,97 \mathrm{~b}$ & $45,40 \mathrm{~b}$ & $13,86 \mathrm{c}$ & $13,86 \mathrm{~b}$ \\
\hline CNA 2042 & $1678 \mathrm{a}$ & $408 \mathrm{~b}$ & $1,06 \mathrm{a}$ & $44,67 \mathrm{~b}$ & $48,14 \mathrm{a}$ & $14,24 \mathrm{c}$ & $15,08 \mathrm{~b}$ \\
\hline CNA 2721 & $1680 \mathrm{a}$ & $482 \mathrm{a}$ & $0,99 \mathrm{a}$ & $31,67 \mathrm{c}$ & $43,03 \mathrm{~b}$ & 34,68 a & $27,93 \mathrm{a}$ \\
\hline CNA 5171 & $1685 a$ & $431 \mathrm{~b}$ & $1,03 \mathrm{a}$ & $46,52 \mathrm{~b}$ & $47,02 \mathrm{~b}$ & $25,61 \mathrm{~b}$ & $16,64 \mathrm{~b}$ \\
\hline CNA 4634 & $1703 \mathrm{a}$ & $727 \mathrm{a}$ & $0,82 \mathrm{~b}$ & $44,34 \mathrm{~b}$ & $43,54 \mathrm{~b}$ & $18,04 \mathrm{c}$ & $29,34 \mathrm{a}$ \\
\hline CNA 4125 & $1721 \mathrm{a}$ & $429 \mathrm{~b}$ & $1,05 \mathrm{a}$ & $46,07 \mathrm{~b}$ & $41,49 \mathrm{~b}$ & $10,43 \mathrm{c}$ & $11,98 \mathrm{~b}$ \\
\hline CNA 3178 & $1756 \mathrm{a}$ & $604 \mathrm{a}$ & $0,92 \mathrm{a}$ & 51,54 a & $51,95 \mathrm{a}$ & $15,72 \mathrm{c}$ & $18,38 \mathrm{~b}$ \\
\hline CNA 4150 & $1761 \mathrm{a}$ & $504 a$ & $1,01 \mathrm{a}$ & 50,38 b & $45,67 \mathrm{~b}$ & $14,89 \mathrm{c}$ & $17,09 \mathrm{~b}$ \\
\hline CNA 1168 & $1841 \mathrm{a}$ & $436 \mathrm{~b}$ & $1,08 \mathrm{a}$ & $47,22 \mathrm{~b}$ & $43,34 \mathrm{~b}$ & $14,20 \mathrm{c}$ & $21,61 \mathrm{a}$ \\
\hline CNA 4178 & $1861 \mathrm{a}$ & $429 \mathrm{~b}$ & $1,08 \mathrm{a}$ & $47,05 \mathrm{~b}$ & $45,65 \mathrm{~b}$ & 20,37 c & $26,77 \mathrm{a}$ \\
\hline CNA 5166 & $1897 \mathrm{a}$ & $268 \mathrm{c}$ & $1,20 \mathrm{a}$ & $55,20 \mathrm{a}$ & $45,57 \mathrm{~b}$ & $15,51 \mathrm{c}$ & $16,47 \mathrm{~b}$ \\
\hline CNA 4181 & $1939 a$ & $507 a$ & $1,04 \mathrm{a}$ & $46,38 \mathrm{~b}$ & 47,89 a & $9,40 \mathrm{c}$ & $14,56 \mathrm{~b}$ \\
\hline CNA 4108 & $1968 \mathrm{a}$ & $662 \mathrm{a}$ & $0,95 \mathrm{a}$ & $45,58 \mathrm{~b}$ & $43,56 \mathrm{~b}$ & $8,06 \mathrm{c}$ & $15,65 \mathrm{~b}$ \\
\hline CNA 4141 & $1988 \mathrm{a}$ & $618 \mathrm{a}$ & $0,96 \mathrm{a}$ & $50,94 \mathrm{~b}$ & $52,36 \mathrm{a}$ & $9,56 \mathrm{c}$ & $16,32 \mathrm{~b}$ \\
\hline CNA 4474 & 1997 a & $546 \mathrm{a}$ & $1,03 \mathrm{a}$ & $47,16 \mathrm{~b}$ & $42,18 \mathrm{~b}$ & $10,19 \mathrm{c}$ & $13,61 \mathrm{~b}$ \\
\hline CNA 1462 & 1998 a & $465 \mathrm{~b}$ & $1,05 \mathrm{a}$ & $50,14 \mathrm{~b}$ & $42,79 \mathrm{~b}$ & $16,84 \mathrm{c}$ & $27,46 \mathrm{a}$ \\
\hline CNA 5164 & 2017 a & $378 \mathrm{~b}$ & $1,15 \mathrm{a}$ & 57,22 a & $45,63 \mathrm{~b}$ & $14,43 \mathrm{c}$ & $18,53 \mathrm{~b}$ \\
\hline CNA 4640 & 2065 a & $606 \mathrm{a}$ & $0,98 \mathrm{a}$ & $43,80 \mathrm{~b}$ & $51,50 \mathrm{a}$ & $18,46 \mathrm{c}$ & $15,57 \mathrm{~b}$ \\
\hline CNA 4164 & 2068 a & $261 c$ & $1,24 \mathrm{a}$ & $54,83 \mathrm{a}$ & 50,59 a & $12,70 \mathrm{c}$ & $8,27 \mathrm{~b}$ \\
\hline CNA 5165 & $2118 \mathrm{a}$ & $440 \mathrm{~b}$ & $1,12 \mathrm{a}$ & $56,58 \mathrm{a}$ & 54,95 a & $10,90 \mathrm{c}$ & $11,95 \mathrm{~b}$ \\
\hline CNA 8521 & $2177 \mathrm{a}$ & $389 \mathrm{~b}$ & $1,16 \mathrm{a}$ & $49,81 \mathrm{~b}$ & $51,95 \mathrm{a}$ & $16,50 \mathrm{c}$ & $18,87 \mathrm{~b}$ \\
\hline CNA 2720 & $2202 \mathrm{a}$ & $417 \mathrm{~b}$ & $1,13 \mathrm{a}$ & $58,91 \mathrm{a}$ & $52,88 \mathrm{a}$ & $14,09 \mathrm{c}$ & $22,51 \mathrm{a}$ \\
\hline CNA 4120 & $2217 \mathrm{a}$ & $650 \mathrm{a}$ & $0,90 \mathrm{a}$ & $47,62 \mathrm{~b}$ & 55,25 a & $11,94 \mathrm{c}$ & 19,72 b \\
\hline CNA 4617 & $2333 \mathrm{a}$ & $330 \mathrm{c}$ & $1,20 \mathrm{a}$ & $61,77 \mathrm{a}$ & $53,42 \mathrm{a}$ & $12,84 \mathrm{c}$ & $11,61 \mathrm{~b}$ \\
\hline CNA 4476 & 2349 a & $658 \mathrm{a}$ & $1,02 \mathrm{a}$ & $55,67 \mathrm{a}$ & $51,01 \mathrm{a}$ & $10,50 \mathrm{c}$ & $24,69 \mathrm{a}$ \\
\hline CNA 4140 & $2509 \mathrm{~A}$ & $440 \mathrm{~b}$ & $1,17 \mathrm{a}$ & $50,19 \mathrm{~b}$ & $43,89 \mathrm{~b}$ & $14,67 \mathrm{c}$ & $20,07 \mathrm{~b}$ \\
\hline
\end{tabular}

${ }^{1}$ Médias seguidas da mesma letra, na coluna, não diferiram significativamente a nível de $5 \%$ de probabilidade, pelo teste de Scott-Knott

2IP - Índice de eficiência do uso de fósforo 
Tabela 3. Matéria seca, massa de 100 grãos, número de grãos por panícula e altura das plantas das linhagens avaliadas para a eficiência ao uso de fósforo ${ }^{1}$

\begin{tabular}{|c|c|c|c|c|c|c|c|c|}
\hline \multirow{2}{*}{ Linhagens } & \multicolumn{2}{|c|}{$\mathrm{MS}^{2}$ (g por planta) } & \multicolumn{2}{|c|}{ Massa de 100 grãos (g) } & \multicolumn{2}{|c|}{ Grãos por panícula $\left(n^{0}\right)$} & \multicolumn{2}{|c|}{ Altura planta (cm) } \\
\hline & C/fósforo & S/fósforo & C/fósforo & S/fósforo & C/fósforo & S/fósforo & C/fósforo & S/fósforo \\
\hline CNA 2826 & $5.230 \mathrm{a}$ & $3.483 \mathrm{a}$ & $1,99 \mathrm{~d}$ & $2,85 \mathrm{a}$ & $117 \mathrm{a}$ & $107 \mathrm{a}$ & $110 \mathrm{a}$ & $91 \mathrm{a}$ \\
\hline CNA 4179 & $1.610 \mathrm{c}$ & $1.597 \mathrm{a}$ & $3,52 \mathrm{a}$ & $2,98 \mathrm{a}$ & $70 \mathrm{~b}$ & 79 a & $81 \mathrm{c}$ & $75 \mathrm{~b}$ \\
\hline CNA 2739 & $1.773 \mathrm{c}$ & $1.353 \mathrm{a}$ & $2,78 \mathrm{~b}$ & $2,42 \mathrm{~b}$ & $99 \mathrm{~b}$ & $53 \mathrm{a}$ & $97 \mathrm{~b}$ & $73 \mathrm{~b}$ \\
\hline CNA 0486 & $2.340 \mathrm{c}$ & $1.900 \mathrm{a}$ & $2,52 \mathrm{c}$ & $2,83 \mathrm{a}$ & $103 \mathrm{~b}$ & $92 \mathrm{a}$ & $95 \mathrm{c}$ & $87 \mathrm{a}$ \\
\hline CNA 2836 & $3.877 \mathrm{a}$ & $2.133 \mathrm{a}$ & $2,55 \mathrm{c}$ & $2,95 \mathrm{a}$ & $111 \mathrm{~b}$ & $97 \mathrm{a}$ & $117 \mathrm{a}$ & $97 \mathrm{a}$ \\
\hline CNA 3373 & $1.213 \mathrm{c}$ & 627 a & $2,30 \mathrm{c}$ & $2,28 \mathrm{~b}$ & $83 \mathrm{~b}$ & $44 \mathrm{a}$ & $57 \mathrm{~d}$ & $41 \mathrm{c}$ \\
\hline CNA 4411 & $3.280 \mathrm{~b}$ & $2.490 \mathrm{a}$ & $2,77 \mathrm{~b}$ & $2,48 \mathrm{~b}$ & $109 \mathrm{~b}$ & $125 \mathrm{a}$ & $88 \mathrm{c}$ & $79 \mathrm{a}$ \\
\hline CNA 0799 & $2.163 \mathrm{c}$ & $1.467 \mathrm{a}$ & $2,29 \mathrm{c}$ & $2,53 \mathrm{~b}$ & $72 \mathrm{~b}$ & $64 \mathrm{a}$ & $86 \mathrm{c}$ & $73 \mathrm{~b}$ \\
\hline CNA 4291 & 3.377 b & $2.263 \mathrm{a}$ & $1,70 \mathrm{~d}$ & $2,13 \mathrm{~b}$ & $141 \mathrm{a}$ & 159 a & $92 \mathrm{c}$ & $84 \mathrm{a}$ \\
\hline CNA 1383 & $3.500 \mathrm{~b}$ & $2.243 \mathrm{a}$ & 3,07 b & $2,90 \mathrm{a}$ & $126 \mathrm{a}$ & $116 \mathrm{a}$ & $106 \mathrm{a}$ & $95 \mathrm{a}$ \\
\hline CNA 5163 & $3.057 \mathrm{~b}$ & $2.513 \mathrm{a}$ & 3,27 a & 3,18 a & $144 \mathrm{a}$ & $104 \mathrm{a}$ & $97 \mathrm{~b}$ & 88 a \\
\hline CNA 2523 & $4.393 \mathrm{a}$ & $2.220 \mathrm{a}$ & $2,49 \mathrm{c}$ & $2,75 \mathrm{~b}$ & $152 a$ & $84 \mathrm{a}$ & $116 \mathrm{a}$ & $87 \mathrm{a}$ \\
\hline CNA 4171 & $2.133 \mathrm{c}$ & $1.917 \mathrm{a}$ & $3,45 \mathrm{a}$ & $3,24 \mathrm{a}$ & $85 \mathrm{~b}$ & $81 \mathrm{a}$ & $89 \mathrm{c}$ & $75 \mathrm{~b}$ \\
\hline CNA 4148 & $4.430 \mathrm{a}$ & $1.887 \mathrm{a}$ & $2,87 \mathrm{~b}$ & $2,65 \mathrm{~b}$ & $133 \mathrm{a}$ & $62 \mathrm{a}$ & $100 \mathrm{~b}$ & $78 \mathrm{~b}$ \\
\hline CNA 6571 & $2.277 \mathrm{c}$ & $1.837 \mathrm{a}$ & $3,18 \mathrm{~b}$ & $2,92 \mathrm{a}$ & $95 \mathrm{~b}$ & $81 \mathrm{a}$ & $93 \mathrm{c}$ & $90 \mathrm{a}$ \\
\hline CNA 3289 & $2.513 \mathrm{c}$ & $1.947 \mathrm{a}$ & $2,96 \mathrm{~b}$ & $2,56 \mathrm{~b}$ & $118 \mathrm{a}$ & $86 \mathrm{a}$ & $78 \mathrm{c}$ & $67 \mathrm{~b}$ \\
\hline CNA 5021 & $1.987 \mathrm{c}$ & $1.827 \mathrm{a}$ & $3,10 \mathrm{~b}$ & $2,91 \mathrm{a}$ & $133 \mathrm{a}$ & $88 \mathrm{a}$ & $94 \mathrm{c}$ & $83 \mathrm{a}$ \\
\hline CNA 5167 & $2.397 \mathrm{c}$ & $1.940 \mathrm{a}$ & $3,50 \mathrm{a}$ & $3,06 \mathrm{a}$ & $76 \mathrm{~b}$ & $73 \mathrm{a}$ & $97 \mathrm{~b}$ & $83 a$ \\
\hline CNA 4105 & $2.060 \mathrm{c}$ & $1.543 \mathrm{a}$ & $3,17 \mathrm{~b}$ & $3,29 \mathrm{a}$ & $95 \mathrm{~b}$ & $51 \mathrm{a}$ & $91 \mathrm{c}$ & $70 \mathrm{~b}$ \\
\hline CNA 6564 & $1.897 \mathrm{c}$ & $2.067 \mathrm{a}$ & $3,10 \mathrm{~b}$ & $3,03 a$ & $101 \mathrm{~b}$ & $76 \mathrm{a}$ & $90 \mathrm{c}$ & $80 a$ \\
\hline CNA 4166 & $2.980 \mathrm{c}$ & $2.043 \mathrm{a}$ & $3,35 \mathrm{a}$ & 3,39 a & $90 \mathrm{~b}$ & $68 \mathrm{a}$ & $98 \mathrm{~b}$ & $81 \mathrm{a}$ \\
\hline CA 780284 & $4.847 \mathrm{a}$ & $2.510 \mathrm{a}$ & $2,93 \mathrm{~b}$ & $2,73 \mathrm{~b}$ & $138 \mathrm{a}$ & $76 \mathrm{a}$ & $104 \mathrm{~b}$ & 81 a \\
\hline CNA 4180 & $2.037 \mathrm{c}$ & $1.700 \mathrm{a}$ & 3,56 a & $3,48 \mathrm{a}$ & $82 \mathrm{~b}$ & $55 \mathrm{a}$ & $90 \mathrm{c}$ & $76 \mathrm{~b}$ \\
\hline CNA 4144 & $4.047 \mathrm{a}$ & $2.400 \mathrm{a}$ & $2,64 \mathrm{c}$ & $2,99 \mathrm{a}$ & $103 \mathrm{~b}$ & $81 \mathrm{a}$ & $97 \mathrm{~b}$ & $80 \mathrm{a}$ \\
\hline CNA 2865 & $3.460 \mathrm{~b}$ & $2.167 \mathrm{a}$ & $2,40 \mathrm{c}$ & $2,81 \mathrm{a}$ & $120 \mathrm{a}$ & $87 \mathrm{a}$ & $108 \mathrm{a}$ & $88 \mathrm{a}$ \\
\hline CNA 6430 & $2.673 \mathrm{c}$ & $1.817 \mathrm{a}$ & $3,05 \mathrm{~b}$ & $2,89 \mathrm{a}$ & $101 \mathrm{~b}$ & $64 \mathrm{a}$ & $96 \mathrm{~b}$ & $75 \mathrm{~b}$ \\
\hline CNA 2042 & $3.587 \mathrm{~b}$ & $2.230 \mathrm{a}$ & $3,10 \mathrm{~b}$ & 3,08 a & $123 \mathrm{a}$ & $88 \mathrm{a}$ & $101 \mathrm{~b}$ & $84 \mathrm{a}$ \\
\hline CNA 2721 & $4.047 \mathrm{a}$ & $2.330 \mathrm{a}$ & $2,54 \mathrm{c}$ & $2,97 \mathrm{a}$ & $129 \mathrm{a}$ & $96 \mathrm{a}$ & $111 \mathrm{a}$ & 88 a \\
\hline CNA 5171 & $3.323 \mathrm{~b}$ & $1.653 \mathrm{a}$ & $3,04 \mathrm{~b}$ & $3,03 \mathrm{a}$ & $125 \mathrm{a}$ & $67 \mathrm{a}$ & $91 \mathrm{c}$ & $68 \mathrm{~b}$ \\
\hline CNA 4634 & $3.847 \mathrm{a}$ & $2.633 \mathrm{a}$ & $2,99 \mathrm{~b}$ & $3,20 \mathrm{a}$ & 139 a & $97 \mathrm{a}$ & $99 \mathrm{~b}$ & $81 \mathrm{a}$ \\
\hline CNA 4125 & $3.020 \mathrm{~b}$ & $3.007 \mathrm{a}$ & $3,05 \mathrm{~b}$ & $3,09 \mathrm{a}$ & $97 \mathrm{~b}$ & $80 \mathrm{a}$ & $102 \mathrm{~b}$ & $81 \mathrm{a}$ \\
\hline CNA 3178 & $3.970 \mathrm{a}$ & $2.480 \mathrm{a}$ & $3,14 \mathrm{~b}$ & 3,07 a & $143 \mathrm{a}$ & $111 \mathrm{a}$ & $103 \mathrm{~b}$ & 84 a \\
\hline CNA 4150 & $3.093 \mathrm{~b}$ & $2.480 \mathrm{a}$ & $3,01 \mathrm{~b}$ & $2,92 \mathrm{a}$ & $97 \mathrm{~b}$ & $92 \mathrm{a}$ & $102 \mathrm{~b}$ & $86 \mathrm{a}$ \\
\hline CNA 1168 & $3.783 \mathrm{a}$ & $1.993 \mathrm{a}$ & 3,27 a & $3,20 \mathrm{a}$ & $143 \mathrm{a}$ & $72 \mathrm{a}$ & $98 \mathrm{~b}$ & $80 \mathrm{a}$ \\
\hline CNA 4178 & $3.837 \mathrm{a}$ & $2.080 \mathrm{a}$ & $3,12 \mathrm{~b}$ & $2,87 \mathrm{a}$ & $153 \mathrm{a}$ & $91 \mathrm{a}$ & $105 \mathrm{a}$ & $81 \mathrm{a}$ \\
\hline CNA 5166 & $2.583 \mathrm{c}$ & $1.917 \mathrm{a}$ & $3,60 \mathrm{a}$ & 3,18 a & $106 \mathrm{~b}$ & $74 \mathrm{a}$ & $98 \mathrm{~b}$ & $76 \mathrm{~b}$ \\
\hline CNA 4181 & $3.827 \mathrm{a}$ & $2.417 \mathrm{a}$ & $3,08 \mathrm{~b}$ & 3,49 a & $128 \mathrm{a}$ & $92 \mathrm{a}$ & $106 \mathrm{a}$ & $82 \mathrm{a}$ \\
\hline CNA 4108 & $2.873 \mathrm{c}$ & $1.933 \mathrm{a}$ & $3,09 \mathrm{~b}$ & $2,93 \mathrm{a}$ & $85 \mathrm{~b}$ & $71 \mathrm{a}$ & $98 \mathrm{~b}$ & 86 a \\
\hline CNA 4141 & $2.880 \mathrm{c}$ & $2.057 \mathrm{a}$ & 3,27 a & $3,10 \mathrm{a}$ & $102 \mathrm{~b}$ & $95 \mathrm{a}$ & $86 \mathrm{c}$ & $77 \mathrm{~b}$ \\
\hline CNA 4474 & $3.193 \mathrm{~b}$ & $1.963 \mathrm{a}$ & $2,24 \mathrm{c}$ & $2,19 \mathrm{~b}$ & $153 \mathrm{a}$ & $86 \mathrm{a}$ & $100 \mathrm{~b}$ & $78 \mathrm{~b}$ \\
\hline CNA 1462 & $3.137 \mathrm{~b}$ & $1.863 \mathrm{a}$ & $3,56 \mathrm{a}$ & $3,14 \mathrm{a}$ & $123 \mathrm{a}$ & $67 \mathrm{a}$ & $90 \mathrm{c}$ & $68 \mathrm{~b}$ \\
\hline CNA 5164 & $2.120 \mathrm{c}$ & $1.747 \mathrm{a}$ & 3,31 a & 3,03 a & $106 \mathrm{~b}$ & $72 \mathrm{a}$ & $94 \mathrm{c}$ & $74 \mathrm{~b}$ \\
\hline CNA 4640 & $3.850 \mathrm{a}$ & $2.057 \mathrm{a}$ & $3,00 \mathrm{~b}$ & $3,16 \mathrm{a}$ & $128 \mathrm{a}$ & $92 \mathrm{a}$ & $98 \mathrm{~b}$ & $79 \mathrm{a}$ \\
\hline CNA 4164 & $2.100 \mathrm{c}$ & $1.687 \mathrm{a}$ & 3,49 a & $3,03 \mathrm{a}$ & $93 \mathrm{~b}$ & $63 \mathrm{a}$ & $90 \mathrm{c}$ & $76 \mathrm{~b}$ \\
\hline CNA 5165 & $2.307 \mathrm{c}$ & $1.490 \mathrm{a}$ & $3,50 \mathrm{a}$ & $3,44 \mathrm{a}$ & $101 \mathrm{~b}$ & $65 \mathrm{a}$ & $94 \mathrm{c}$ & $75 \mathrm{~b}$ \\
\hline CNA 8521 & $3.190 \mathrm{~b}$ & $1.833 \mathrm{a}$ & $2,92 \mathrm{~b}$ & $3,41 \mathrm{a}$ & $108 \mathrm{~b}$ & $82 \mathrm{a}$ & $103 \mathrm{~b}$ & $84 \mathrm{a}$ \\
\hline CNA 2720 & $2.747 \mathrm{c}$ & $1.990 \mathrm{a}$ & $3,23 \mathrm{a}$ & $3,04 \mathrm{a}$ & $143 \mathrm{a}$ & $103 a$ & $112 \mathrm{a}$ & $91 \mathrm{a}$ \\
\hline CNA 4120 & $3.760 \mathrm{a}$ & $1.917 \mathrm{a}$ & $3,09 \mathrm{~b}$ & $3,29 a$ & $135 \mathrm{a}$ & 79 a & $112 \mathrm{a}$ & $86 \mathrm{a}$ \\
\hline CNA 4617 & $2.207 \mathrm{c}$ & $1.533 \mathrm{a}$ & $3,08 \mathrm{~b}$ & $2,96 \mathrm{a}$ & $127 \mathrm{a}$ & $81 \mathrm{a}$ & $85 \mathrm{c}$ & $68 \mathrm{~b}$ \\
\hline CNA 4476 & $2.430 \mathrm{c}$ & $1.990 \mathrm{a}$ & $3,00 \mathrm{~b}$ & $2,99 \mathrm{a}$ & $108 \mathrm{~b}$ & $91 \mathrm{a}$ & $97 \mathrm{~b}$ & $82 \mathrm{a}$ \\
\hline CNA 4140 & $4.073 \mathrm{a}$ & $2.097 \mathrm{a}$ & $3,26 \mathrm{a}$ & $3,00 \mathrm{a}$ & $159 \mathrm{a}$ & $81 \mathrm{a}$ & $103 \mathrm{~b}$ & $77 \mathrm{~b}$ \\
\hline
\end{tabular}

${ }^{1}$ Médias seguidas da mesma letra, na coluna, não diferiram significativamente a nível de $5 \%$ de probabilidade, pelo teste de Scott-Knott

${ }^{2} \mathrm{MS}$ - Matéria seca da parte aérea das plantas 
Tabela 4. Coeficiente de correlação entre as variáveis avaliadas: produtividade, matéria seca das plantas (MS), índice de colheita (IC), matéria seca de 100 grãos (MS100), esterilidade de espiguetas (EE), número de grãos por panícula (GPAN) e altura das plantas, em condições de baixa disponibilidade de fósforo no solo, e índice de eficiência do uso de fósforo (IP), tomando-se como referência a produtividade de grãos

\begin{tabular}{|c|c|c|c|c|c|c|c|}
\hline & $\begin{array}{l}\text { Produtividade } \\
\left(\mathrm{kg} \mathrm{ha}^{-1}\right)\end{array}$ & $\begin{array}{c}\text { MS } \\
\left(\mathrm{kg} \mathrm{ha}^{-1}\right)\end{array}$ & IC & $\begin{array}{l}\text { MS100 } \\
\text { (g) }\end{array}$ & $\begin{array}{l}\mathrm{EE} \\
(\%)\end{array}$ & GPAN & $\begin{array}{l}\text { Altura } \\
\text { (cm) }\end{array}$ \\
\hline \multirow{2}{*}{ MS $\left(\mathrm{kg} \mathrm{ha}^{-1}\right)$} & 0,267 & & & & & & \\
\hline & 0,058 & & & & & & \\
\hline \multirow{2}{*}{ IC } & 0,130 & $-0,414$ & & & & & \\
\hline & 0,364 & 0,002 & & & & & \\
\hline \multirow{2}{*}{ MS100 (g) } & $-0,034$ & 0,111 & 0,133 & & & & \\
\hline & 0,810 & 0,437 & 0,353 & & & & \\
\hline \multirow{2}{*}{ EE (\%) } & 0,317 & 0,409 & $-0,369$ & $-0,120$ & & & \\
\hline & 0,024 & 0,003 & 0,008 & 0,401 & & & \\
\hline \multirow{2}{*}{ GPAN } & 0,439 & 0,618 & 0,125 & $-0,222$ & 0,353 & & \\
\hline & 0,001 & $<0,0001$ & 0,382 & 0,118 & 0,011 & & \\
\hline \multirow{2}{*}{ Altura $(\mathrm{cm})$} & 0,312 & 0,650 & $-0,2334$ & 0,193 & 0,282 & 0,572 & \\
\hline & 0,026 & $<0,0001$ & 0,098 & 0,176 & 0,045 & $<0,0001$ & \\
\hline \multirow{2}{*}{ IP } & $-0,626$ & $-0,241$ & 0,045 & 0,384 & $-0,405$ & $-0,474$ & $-0,216$ \\
\hline & $<0,0001$ & 0,089 & 0,756 & 0,005 & 0,003 & $<0,0001$ & 0,127 \\
\hline
\end{tabular}

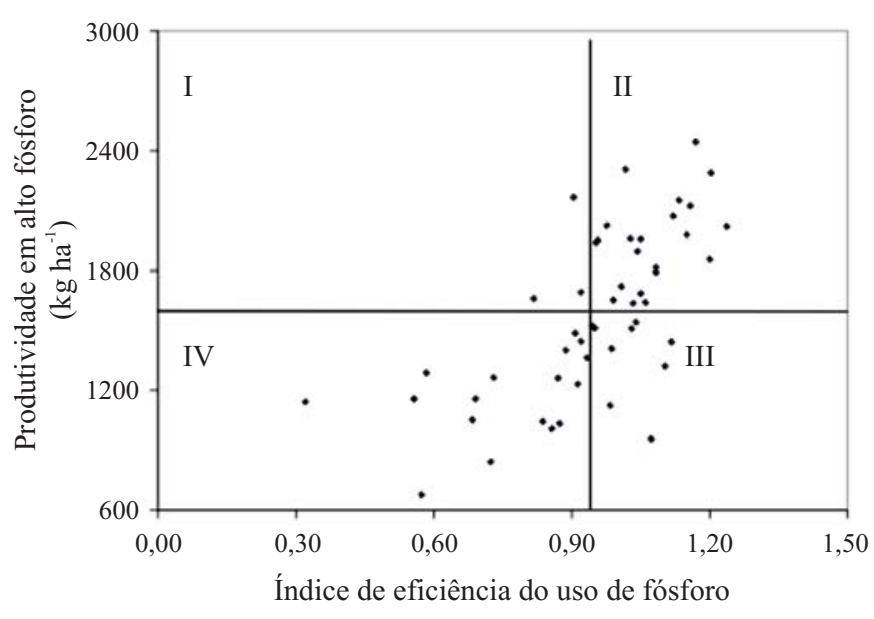

Figura 1. Distribuição das linhagens em quartis delimitados pelo índice de eficiência do uso de fósforo e pela produtividade de grãos no tratamento sem deficiência de fósforo no solo, nos pontos determinados por suas médias

\section{CONCLUSÕES}

1. O índice de eficiência do uso de fósforo mostrou ser mais adequado que a produtividade de grãos em baixo teor de fósforo no solo na avaliação da eficiência diferenciada das linhagens no uso do $\mathrm{P}$.

2. As linhagens CNA 4634, CNA 4120 e CNA 3178 apresentam bom comportamento produtivo tanto em alto como em baixo teor de P no solo, enquanto as CNA 4108, CNA 4141, CNA 4640, CNA 2721, CNA 4150, CNA 4476, CNA 4474, CNA 5171, CNA 4181, CNA 1462, CNA 4125, CNA 2042, CNA 1168, CNA 4178, CNA 5165, CNA 2720, CNA 5164, CNA 8521, CNA 4140, CNA 5166, CNA 4617 e CNA 4164, indicaram bom comportamento produtivo em alto teor de P no solo; entretanto, não são eficientes no aproveitamento de baixo P disponível no solo.

\section{LITERATURA CITADA}

Aboulroos, S. A.; Nielsen, N. E. Effects of phosphorus placement in varying, confined volumes of soil on fineness and density of barley roots and the mean rate of nutrient uptake per root unit. Acta Agriculturae Scandinavica, Stockholm, v.29, n.4, p.331-336, 1979.

Claessen, M. E. C. (Org.). Manual de métodos de análise de solo. 2.ed. rev. atual. Rio de Janeiro: Embrapa-CNPS, 1997. 212p. (Documentos, 1)

Dobermann, A.; Cassman, K. G.; Mamaril, C. P.; Sheehy, J. E. Management of phosphorus, potassium, and sulfur in intensive, irrigated lowland rice. Field Crops Research, Amsterdam, v.56, p.113-138, 1998.

Eissenstar, D. M. Costs and benefits of construction roots of small diameter. Journal of Plant Nutrition, New York, v.15, p.763-782, 1992.

Fageria, N. K. Tolerância diferencial de cultivares de arroz ao alumínio em solução nutritiva. Pesquisa Agropecuária Brasileira, Brasília, v.17, n.1, p.1-9, 1982.

Fageria, N. K. Nutrição mineral. In: Vieira, N. R. de A.; Santos, A. B. dos; Sant'Ana, E. P. (eds.). A cultura do arroz no Brasil. Santo Antônio de Goiás: Embrapa Arroz e Feijão, 1999. p.172-196.

Fageria, N. K.; Baligar, V. C. Upland rice genotypes evaluation for phosphorus use efficiency. Journal of Plant Nutrition, New York, v.20, n.4/5, p.499-509, 1997.

Fisher, R. A.; Maurer, R. Drought resistance in spring wheat cultivars. I. Grain yield responses. Journal of Agricultural Research, Victoria, v.29, p.897-912, 1978.

Gahoonia, T. S.; Nielsen N. E. Variation in root hairs of barley cultivars doubled soil phosphorus uptake. Euphytica, Wageningen, v.98, p.177-182, 1997.

Gourley, C. J. P.; Allan, D. L.; Russelle, M. P. Defining phosphorus efficiency in plants. Plant and Soil, The Hague, v.156, p.289-292, 1993. 
Haynes, R. J.; Ludecke, T. E. Yield, root morphology and chemical composition of two legumes as affected by lime and phosphorus applications to an acid soil. Plant and Soil, The Hague, v.62, p.241-254, 1981

Horst, W. J.; Kamh, M.; Jibrin, J. M.; Chude, V. O. Agronomic measures for increasing $\mathrm{P}$ availability to crops. Plant and Soil, The Hague, v.237, p.211-223, 2001.

Lynch, J. P.; Brown, K. M. Topsoil foraging: an architectural adaptation of plants to low phosphorus availability. Plant and Soil, The Hague, v.237, p.225-237, 2001.

Otani, T.; Ae, N. Sensitivity of phosphorus uptake to change in root length and soil volume. Agronomy Journal, Madison, v.88, p.371-375, 1996.

Sanchez, P. A.; Salinas, J. G. Low-input technology for managing oxisols and ultisols in tropical America. Advances in Agronomy, New York, v.34, p.279-406, 1981.
Sattelmacher, B.; Horst, W. J.; Becker, H. C. Factors that contribute to genetic variation for nutrient efficiency of crop plants. Zeitschrift fur Pflanzenernaehrung und Bodenkunde, Berlin, v.157, p.215-224, 1994.

Wissuwa, M. How do plants achieve tolerance to phosphorus deficiency - small causes with big effects. Plant Physiology, Bethesda, v.133, p.1947-1958, 2003.

Wissuwa, M. Combining a modeling with a genetic approach in establishing associations between genetic and physiological effects in relation to phosphorus uptake. Plant and Soil, The Hague, v.269, n.1/2, p.57-68, 2005.

Wissuwa, M.; Ae, N. Genotypic variation for tolerance to phosphorus deficiency in rice and the potential for its exploitation in rice improvement, Plant Breeding, The Hague, v.120, p.43-48, 2001. 